\title{
Avaliação de coberturas mortas em cultura de alface sob manejo orgânico
}

\author{
Fabio F de Oliveira ${ }^{1}$; José Guilherme M Guerra²; Dejair L de Almeida ${ }^{3}$; Raul de LD Ribeiro ${ }^{4}$; José \\ Antonio A Espindola²; Marta dos SF Ricci²; Marcos B Ceddia ${ }^{4}$

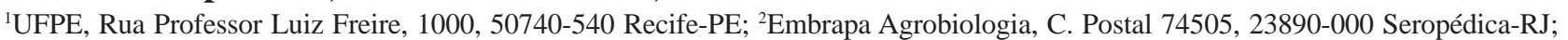 \\ ${ }_{3}^{3}$ Sítio Barra do Santa Teresa, Estrada RJ 116, km 100, 28660-000 Bom Jardim-RJ; ${ }^{4}$ UFRRJ-Instituto de Agronomia, BR 465, km 07, \\ 23851-970 Seropédica-RJ; oliveiraff@yahoo.com.br; gmguerra@cnpab.embrapa.br; jose@ cnpab.embrapa.br; marta@cnpab.embrapa.br; \\ dejair_la@yahoo.com.br; ceddia@ufrrj.br
}

\section{RESUMO}

A cobertura morta do solo com leguminosas e gramíneas é uma prática cultural que traz benefícios aos sistemas de produção. Foi conduzido um experimento no município de Seropédica, estado do Rio de Janeiro, com o objetivo de avaliar o efeito de diferentes tipos de cobertura morta sobre a reinfestação de ervas espontâneas e sobre o desempenho agronômico de alface, em cultivo orgânico. O delineamento experimental adotado foi blocos casualizados com quatro repetições e parcelas de oito plantas na área útil. Os tratamentos foram: bagaço de cana-de-açúcar (Saccharum sp.), bambu (Bambuza sp.), capim Cameroon (Penisetum purpureum), crotalária (Crotalaria juncea), eritrina (Erythrina poeppigiana), gliricídia (Gliricidia sepium), guandu (Cajanus cajan), mucuna cinza (Mucuna pruriens) e controle (sem cobertura dos canteiros). Estimou-se a decomposição in situ e a liberação de nitrogênio de cada cobertura morta. Foram avaliados dois ciclos consecutivos de alface, cultivar Regina, na mesma área, visando a comparar o efeito residual das coberturas mortas. Observou-se maior acúmulo de $\mathrm{N}$ nos resíduos vegetais das leguminosas (máximo de $1.010 \mathrm{~kg} \mathrm{ha}^{-1}$ com mucunacinza). Em relação à decomposição da cobertura morta, avaliada na colheita do primeiro ciclo de alface (35 dias após o transplante), as leguminosas apresentaram percentuais inferiores aos das gramíneas de massa seca e $\mathrm{N}$ remanescentes. A reinfestação dos canteiros pelas ervas espontâneas não diferiu significativamente entre coberturas mortas, variando entre 31 e 58 plantas $\mathrm{m}^{-2}$, mas a redução da densidade populacional da vegetação reinfestante chegou a $83 \%$ em comparação ao tratamento controle. Em ambos os ciclos de cultivo da alface, a massa fresca (de 315,8 a 366,0 e de 202,9 a 225,0 g planta $^{-1}$, respectivamente nos primeiro e segundo ciclos), o diâmetro da cabeça (de 30,8 a 31,7 e de 25,5 a $28,5 \mathrm{~cm}$ ) e o teor de $\mathrm{N}$ (de 32,3 a 38,8 e de 28,0 a $30,3 \mathrm{~g} \mathrm{~kg}^{-1}$ ) foram superiores quando leguminosas foram utilizadas como cobertura morta.

Palavras-chave: Lactuca sativa, cobertura do solo, controle de ervas espontâneas, gramíneas, leguminosas, nitrogênio.

\section{ABSTRACT}

\section{Evaluation of mulches on organically grown lettuce}

Soil mulching with legumes and grasses is an agricultural practice which promotes benefits to production systems. An experiment was carried out at Seropédica, Rio de Janeiro State, to evaluate the effects of mulch types on weed control and agronomic performance of organically grown lettuce. A randomized blocks design was adopted, with four replications and eight plants in the useful area of each plot. The treatments were: sugar cane (Saccharum sp.) bagasse, bamboo (Bambuza sp.), Cameroon grass (Penisetum purpureum), sunn hemp (Crotalaria juncea), mountain immortelle (Erythrina poeppigiana), gliricidia (Gliricidia sepium), pigeon pea (Cajanus cajan), velvet bean (Mucuna pruriens) and control (no mulching). In situ decomposition and nitrogen release rates were estimated for each mulch. Two consecutive cycles of lettuce (cv. Regina) were conducted in the same area to compare residual effects of mulching. There were greater accumulations of $\mathrm{N}$ in the legumes residues (with a maximum of $1.010 \mathrm{~kg} \mathrm{ha}^{-1}$, at velvet bean). Legumes residues showed lower contents of remaining dry matter and $\mathrm{N}$ than grasses, at the end of the first cultivation cycle of lettuce (35 days after transplanting). Weed populations did not differ in relation to the mulch source, varying from 31 to 58 plants $\mathrm{m}^{-2}$. The reduction of weed infestation reached $83 \%$ as compared to the control treatment. In both crop cycles, lettuce shoot dry matter (315.8 to 366.0, and 202.9 to 225.0 g plant $^{-1}$, respectively at the first and the second cultivation cycles), diameter ( 30.8 to 31.7 , and 25.5 to $28.5 \mathrm{~cm}$ ) and $\mathrm{N}$ content ( 32.3 to 38.8 , and 28.0 to $30.3 \mathrm{~g} \mathrm{~kg}^{-1}$ ) were greater in the treatments using legume mulches.

Keywords: Lactuca sativa, soil cover, weeds control, grasses, legumes, nitrogen.

\section{(Recebido para publicação em 12 de junho de 2007; aceito em 9 de junho de 2008)}

\begin{abstract}
A cobertura morta do solo é uma prática cultural que traz reconhecidos benefícios aos sistemas de produção, especialmente no que diz respeito à olericultura. Dentre as vantagens decorrentes de sua utilização podem ser destacadas, quanto aos atributos do solo, a melhoria da estrutura (Corrêa, 2002), a prevenção à erosão (Smolikowski et al., 2001) e o aporte de matéria orgânica e nutrientes (Cadavid et al., 1998). O po-
\end{abstract}

tencial de controle de ervas espontâneas tem sido também registrado (MacLean et al., 2003).

Diversos fatores influenciam a decomposição de resíduos vegetais introduzidos em áreas de cultivo, tais como a qualidade e abundância da biota do solo, as características específicas do próprio material orgânico e as condições edafoclimáticas de cada região (Correia $\&$ Andrade, 1999). De acordo com es- tes autores, sob idênticas condições de clima e solo, a velocidade de decomposição dos resíduos e a conseqüente liberação de nutrientes são influenciadas pela composição química do material empregado.

Dentre as espécies utilizadas para cobertura morta do solo, destacam-se representantes de leguminosas e gramíneas. As primeiras, pela capacidade de se associarem às bactérias 
fixadoras de nitrogênio, possibilitam elevado aporte desse elemento aos sistemas de produção (Barradas et al., 2001; Padovan et al., 2002). De maneira geral, a palhada de leguminosas, fragmentada e depositada na superfície do solo, caracteriza-se por uma rápida decomposição e liberação de nutrientes (Aita \& Giacomini, 2003), o que tende a favorecer o desempenho agronômico das culturas. Por outro lado, as gramíneas normalmente apresentam decomposição mais lenta, podendo inclusive acarretar imobilização de nutrientes no solo (Espindola et al., 2006).

O objetivo deste estudo foi avaliar a influência de diferentes coberturas mortas sobre a reinfestação pela vegetação espontânea e sobre o desempenho agronômico da cultura da alface, em dois cultivos consecutivos sob manejo orgânico, além de determinar a decomposição e a liberação de $\mathrm{N}$ pelos materiais vegetais avaliados.

\section{MATERIAL E MÉTODOS}

$\mathrm{O}$ experimento foi conduzido em área pertencente ao Sistema Integrado de Produção Agroecológica (Fazendinha Agroecológica Km 47), localizado no município de Seropédica (Baixada Fluminense), estado do Rio de Janeiro. O clima da região pertence à classe Aw, segundo Köppen. O solo onde foi instalado o experimento é classificado como Planossolo, cuja análise química, procedida de acordo com a metodologia preconizada pela Embrapa (1997), apresentou os resultados (camada de $0-20 \mathrm{~cm}): \mathrm{pH}$ em água $=6,1 ; \mathrm{Al}=$ $0,0 \mathrm{mmol}_{\mathrm{c}} \mathrm{dm}^{-3} ; \mathrm{Ca}=13 \mathrm{mmol}_{\mathrm{c}} \mathrm{dm}^{-3}$; $\mathrm{Mg}=7 \mathrm{mmol}_{\mathrm{c}} \mathrm{dm}^{-3} ; \mathrm{P}=21 \mathrm{mg} \mathrm{dm}^{-3}$ e K $=53 \mathrm{mg} \mathrm{dm}^{-3}$.

O preparo do solo consistiu inicialmente de aração e gradagem. Em seguida, os canteiros foram levantados com auxílio de microtrator e enxada rotativa. A adubação desses canteiros, precedendo o transplantio das mudas de alface, correspondeu a 9,1 t ha-1 de esterco bovino (dose equivalente a $100 \mathrm{~kg}$ de $\mathrm{N}$ $\mathrm{ha}^{-1}$ ), além de $30 \mathrm{~kg}$ de $\mathrm{P}$ ha ${ }^{-1}$ e de $60 \mathrm{~kg}$ de $\mathrm{K} \mathrm{ha}^{-1}$, nas formas de termofosfato e sulfato de potássio, respectivamente. A adubação de cobertura com cama-deaviário (teor de $3 \mathrm{~g} \mathrm{~N} \mathrm{~kg}^{-1}$ ) foi realizada

Tabela 1. Características químicas e aporte de nitrogênio de diferentes resíduos vegetais usados para cobertura morta do solo (chemical properties and nitrogen amounts of different residues used for soil mulching). Seropédica, SIPA, 2004.

\begin{tabular}{|c|c|c|c|c|c|c|}
\hline \multirow{2}{*}{ Resíduo } & \multirow{2}{*}{$\begin{array}{c}N \\
\left(g^{\prime} g^{-1}\right)\end{array}$} & \multirow{2}{*}{$\mathrm{C} / \mathrm{N}$} & Lig $^{1}$ & $\mathrm{Pol}^{2}$ & \multirow{2}{*}{$\begin{array}{l}(\mathrm{Lig}+ \\
\mathrm{Pol}) / \mathrm{N}\end{array}$} & \multirow{2}{*}{$\begin{array}{c}\mathrm{N}^{3} \\
\left(\mathrm{~kg} \mathrm{ha}^{-1}\right)\end{array}$} \\
\hline & & & \multicolumn{2}{|c|}{$\left(\mathrm{g} \mathrm{kg}^{-1}\right)$} & & \\
\hline Cana-de-açúcar (S. officinarum) & 3,2 & 131 & 47 & 19 & 20,6 & 89,6 \\
\hline Bambu (Bambuza sp.) & 11,8 & 36 & 53 & 14 & 5,7 & 295,0 \\
\hline Capim Cameroon (P. purpureum) & 6,1 & 69 & 72 & 20 & 15,1 & 152,5 \\
\hline Crotalária (C. juncea) & 27,9 & 15 & 81 & 41 & 4,4 & 641,7 \\
\hline Eritrina (E. poeppigiana) & 35,2 & 12 & 74 & 27 & 2,9 & 880,0 \\
\hline Gliricídia (G. sepium) & 35,1 & 12 & 68 & 18 & 2,5 & 807,3 \\
\hline Guandu (C. cajan) & 21,8 & 19 & 86 & 16 & 4,7 & 545,0 \\
\hline Mucuna cinza (M. pruriens) & 40,4 & 11 & 101 & 25 & 3,1 & 1010,0 \\
\hline
\end{tabular}

${ }^{1} \mathrm{Lig}=$ lignina $\left(\right.$ lignin) $;{ }^{2} \mathrm{Pol}=$ polifenóis (polyphenols); ${ }^{3} \mathrm{~N}=$ quantidade de nitrogênio acumulado nos resíduos vegetais (ammounts of nitrogen accumulated in the residues).

quinze dias após o transplantio das mudas de alface, na dose de $40 \mathrm{~g}_{\text {planta }}{ }^{-1}$.

$\mathrm{O}$ delineamento experimental adotado foi blocos casualizados, com quatro repetições. Os tratamentos consistiram das fontes de cobertura morta: bagaço de cana-de-açúcar (Saccharum sp.), bambu (Bambuza sp.), capim Cameroon (Penisetum purpureum), crotalária (Crotalaria juncea), eritrina (Erythrina poeppigiana), gliricídia (Gliricidia sepium), guandu (Cajanus cajan), mucuna cinza (Mucuna pruriens), além de um tratamento controle, sem cobertura. Os resíduos empregados corresponderam à parte aérea das leguminosas herbáceas (crotalária, guandu e mucuna cinza) e do capim Cameroon, a folhas e ramos finos das leguminosas arbóreas (eritrina e gliricídia), às folhas secas coletadas sob bambuzal e ao bagaço de cana-de-açúcar, recolhido em estabelecimento comercial, após a moagem. Os resíduos foram triturados e, posteriormente, secos à sombra. Utilizou-se, de cada cobertura morta, a quantidade necessária para formar uma camada uniforme e com espessura de 5,0 cm sobre os canteiros.

A aplicação das coberturas mortas foi realizada imediatamente após o preparo dos canteiros. Amostras foram coletadas para estimativas dos teores de nitrogênio, assim como de indicadores da qualidade dos resíduos (relações $\mathrm{C}$ / $\mathrm{N}$, teores de polifenóis e lignina) (Tabela 1). Para tanto, essas amostras foram secas em estufa por 72 horas, até massa constante, e, posteriormente, moídas. A determinação de nitrogênio baseou-se no método recomendado por Bremner \& Mulvaney (1982), o carbono foi determinado pela queima em mufla à temperatura de $550^{\circ} \mathrm{C}$ (Embrapa, 1997) e a análise de polifenóis foi procedida em extratos metanólicos, de acordo com Anderson \& Ingram (1989). As análises de lignina, por sua vez, foram realizadas a partir do método de fibra em detergente ácido (Van Soest \& Wine, 1968).

Para se avaliar a decomposição in situ e a liberação de nitrogênio, amostras de 25 g de cada material foram acondicionadas em sacolas de polietileno de 40 x $30 \mathrm{~cm}$, com malha de $2 \mathrm{~mm}$. Essas sacolas foram distribuídas na superfície das parcelas experimentais quando do transplantio da alface, sendo ali mantidas até a colheita. Nessa ocasião, quantificou-se o material remanescente em relação aos percentuais de massa seca e de N, conforme metodologias já referidas. Para tanto, tomou-se o cuidado de separar partículas de solo aderidas aos resíduos vegetais.

Mudas de alface, cultivar Regina, foram produzidas em casa-de-vegetação, utilizando-se bandejas de isopor com 200 unidades, abastecidas com substrato constituído de subsolo argiloso, esterco bovino curtido, vermicomposto e cama-de-aviário, na proporção respectiva de 8:4:2:1 (base em volume). Três semanas após a semeadura, as mudas de alface foram transplantadas, adotando-se o espaçamento de 0,25 x 0,25 m. A parcela experimental foi constituída de 32 
Tabela 2. Massa seca e nitrogênio remanescentes em diferentes resíduos vegetais utilizados para cobertura morta do solo, após 35 dias de cultivo de alface (remaining dry matter and nitrogen of different residues used for soil mulching, after 35 days of lettuce cultivation). Seropédica, SIPA, 2004.

\begin{tabular}{lcc}
\hline Resíduo & $\begin{array}{c}\text { Massa seca } \\
\text { remanescente (\%) }\end{array}$ & N remanescente (\%) \\
\hline Cana-de-açúcar (S. officinarum) & $73,7 \mathrm{~b}^{1}$ & $68,7 \mathrm{~b}$ \\
Bambu (Bambuza sp.) & $82,3 \mathrm{a}$ & $83,9 \mathrm{a}$ \\
Capim Cameroon (P. purpureum) & $85,2 \mathrm{a}$ & $83,3 \mathrm{a}$ \\
Crotalária (C. juncea) & $49,3 \mathrm{c}$ & $32,7 \mathrm{~d}$ \\
Eritrina (E. poeppigiana) & $52,3 \mathrm{c}$ & $37,1 \mathrm{~d}$ \\
Giricídia (G. sepium) & $51,4 \mathrm{c}$ & $41,9 \mathrm{c}$ \\
Guandu (C. cajan) & $70,1 \mathrm{~b}$ & $61,1 \mathrm{~b}$ \\
Mucuna cinza (M. pruriens) & $51,7 \mathrm{c}$ & $47,4 \mathrm{c}$ \\
\hline
\end{tabular}

${ }^{1}$ Médias seguidas da mesma letra, nas colunas, não diferem entre si pelo teste de Scott-Knott $(\mathrm{p}<0,05)$ (means followed by the same letter, in the column, do not differ from each other by Scott-Knott test, $\mathrm{p}<0.05)$.

plantas de alface, perfazendo uma área de 2,0 $\mathrm{m}^{2}$. A área útil foi representada por quatro plantas ocupando as duas fileiras interiores em cada canteiro, desprezando-se aquelas do início e do final das linhas de plantio. A alface foi colhida 35 dias após o transplantio, amostrando-se as plantas da área útil para determinação da massa fresca e diâmetro da parte aérea (cabeça). Avaliou-se, ainda, o teor de $\mathrm{N}$ nas folhas da alface colhida em cada tratamento. Imediatamente antes da colheita da alface, a população de ervas espontâneas foi estimada através de amostragem feita com o auxílio de moldura de madeira, representando um quadrilátero com área interna de $0,25 \mathrm{~m}^{2}$, colocado no centro de cada parcela. Por ocasião da colheita da alface realizou-se então uma capina manual dos canteiros.

Logo após a colheita da alface, iniciou-se um segundo ciclo, com a mesma cultivar, transplantando-se as mudas para os canteiros ainda cobertos com a palhada remanescente de cada tratamento. Foi efetuada apenas uma adubação de cobertura, 15 dias após o transplantio, aplicando-se $40 \mathrm{~g}$ de cama-de-aviário planta $^{-1}$ sobre a superfície do solo. As características avaliadas foram as mesmas observadas no primeiro ciclo da alface.

Os dados obtidos foram submetidos à análise de variância, através do teste F, comparando-se as médias pelo teste de Scott-Knott. Objetivando detectar possíveis relações entre componentes químicos dos resíduos vegetais e valores remanescentes de massa seca e de $\mathrm{N}$, efetuaram-se testes de correlação de Pearson.

\section{RESULTADOS E DISCUSSÃO}

As fontes de cobertura morta avaliadas apresentaram diferentes comportamentos quanto ao acúmulo de nitrogênio nos resíduos vegetais. Os maiores valores para essa característica mostraram-se associados às leguminosas. No caso da mucuna cinza, por exemplo, verificou-se uma quantidade acumulada da ordem de $1000 \mathrm{~kg}$ de $\mathrm{N} \mathrm{ha}^{-1}$. Por outro lado, coberturas mortas derivadas de gramíneas apresentaram menores quantidades acumuladas desse elemento nos resíduos vegetais, com o máximo de $295 \mathrm{~kg}$ de $\mathrm{N} \mathrm{ha}^{-1}$, correspondente às folhas de bambu (Tabela 1).

A permanência da cobertura no solo e o fornecimento de nitrogênio pelos resíduos vegetais foram avaliados através da determinação dos percentuais de massa seca e de nitrogênio remanescentes após 35 dias do cultivo de alface. De maneira geral, constataram-se percentuais inferiores de massa seca remanescente para as leguminosas, com exceção do guandu (Tabela 2). De forma análoga, os baixos teores de $\mathrm{N}$ remanescente associados aos resíduos de leguminosas indicam ter havido liberação mais rápida a partir dos resíduos desses materiais, o que ocorreu durante o primeiro ciclo de cultivo da alface.
Quanto às características avaliadas nas coberturas mortas nesse estudo, pôde-se observar que as relações $\mathrm{C} / \mathrm{N}$ foram mais baixas nas leguminosas que nas gramíneas (Tabela 1). Dentre as leguminosas, apenas o guandu apresentou alta relação $\mathrm{C} / \mathrm{N}$, o que se explica pelo fato das plantas terem sido cortadas após a fase de florescimento. Por sua vez, as relações (lignina + polifenóis)/ $\mathrm{N}$ foram mais altas para bagaço de canade-açúcar e capim Cameroon. De fato, foi possível estabelecer uma relação significativa entre os teores de $\mathrm{N}$ e as taxas (lignina + polifenóis) $/ \mathrm{N}$ com os percentuais de massa seca remanescente dos resíduos vegetais $(\mathrm{r}=-0,89 * *$ e $0,65^{*}$, respectivamente). Além disso, os teores de $\mathrm{N}$ nos materiais empregados como coberturas mortas também se relacionaram à sua liberação $\left(\mathrm{r}=0,84^{* *}\right)$. Esses resultados encontram respaldo em outros autores, que ressaltam a importância dos teores de N (Constantinides \& Fownes, 1994) e das relações (lignina + polifenóis)/N (Matta-Machado et al., 1994; McDonagh et al., 1995) na liberação desse nutriente a partir de resíduos vegetais em decomposição.

Nos canteiros de alface, a espécie predominante dentre as ervas espontâneas foi Cyperus rotundus (tiririca). As avaliações efetuadas ao final do primeiro ciclo de cultivo da hortaliça indicaram que todas as coberturas mortas foram eficientes no controle da vegetação espontânea reinfestante. De maneira geral, a reinfestação espontânea variou entre 31 e 58 plantas $\mathrm{m}^{-2}$, sem apresentar diferenças significativas entre as coberturas mortas (média de 43 plantas $\mathrm{m}^{-2}$ ), mas mostrando-se significativamente inferior ao valor observado no tratamento controle (189 plantas $\left.\mathrm{m}^{-2}\right)$. Outros autores têm evidenciado o potencial de controle de ervas espontâneas através da adição de resíduos de gramíneas (Correia \& Durigan, 2004) e de leguminosas (Erasmo et al., 2004) à superfície do solo. Esses resultados são geralmente associados a efeitos físicos e químicos. Assim, a cobertura morta é capaz de alterar umidade, luminosidade e temperatura superficial do solo, dificultando a quebra de dormência e a germinação de sementes, além de se constituir em barreira mecânica (Constantin, 
2001). Do ponto de vista químico, os resíduos vegetais podem liberar substâncias capazes de inibir ou retardar a germinação e o crescimento das plântulas, processo este conhecido como alelopatia (Pires \& Oliveira, 2001).

Em ambos os ciclos de cultivo da alface, foram constatados valores mais elevados de massa fresca e diâmetro da parte aérea colhida (cabeça) com as coberturas mortas de leguminosas, à exceção do guandu no primeiro ciclo (Tabela 3). Além disso, as alfaces que compunham os tratamentos com leguminosas apresentaram maior teor de $\mathrm{N}$ (Tabela 3), o que pode ser atribuído à suplementação deste nutriente derivado das leguminosas, conforme anteriormente discutido. Em consonância, Ambrosano et al. (2003) demonstraram a capacidade de leguminosas em contribuir para o aumento do teor de nitrogênio do solo. De acordo com esses autores, $\mathrm{o} \mathrm{N}$ acumulado pelas leguminosas é prontamente mineralizado durante a decomposição de seus resíduos e preservado na matéria orgânica do solo.

Merecem destaque os efeitos proporcionados pelas coberturas mortas de guandu e bambu no decorrer do segundo ciclo de cultivo da alface. A composição química do guandu (baixa relação $\mathrm{C} / \mathrm{N}$ e altos teores de lignina e polifenóis) não permitiu influência positiva da palhada depositada quanto ao crescimento da alface e acúmulo de nitrogênio nos tecidos foliares durante o primeiro ciclo. A subseqüente decomposição dos resíduos do guandu, por ocasião do segundo cultivo da alface, possibilitou a absorção do $\mathrm{N}$ liberado $\mathrm{e}$ o conseqüente estímulo ao desenvolvimento vegetativo da hortaliça.

De forma similar, a palhada de bambu também possibilitou aumento no desempenho da alface durante o segundo ciclo de cultivo. Considerando-se que este aumento do desempenho não foi acompanhado pela elevação dos teores de $\mathrm{N}$ na alface, é possível que tal resultado esteja relacionado a outros benefícios associados à utilização daqueles resíduos como cobertura morta. Gliessman (2001) indica que as coberturas mortas formadas por resíduos de lenta decomposição, como é o caso das gramíneas, contribuem para a conservação da umidade do solo, tendo efeito direto sobre a produção agrí-

Tabela 3. Diâmetro, massa fresca e teor de $\mathrm{N}$ de alface colhida em sistema orgânico de produção, utilizando diferentes coberturas mortas (Diameter, dry matter and $\mathrm{N}$ content of organically grown lettuce). Seropédica, SIPA, 2004.

\begin{tabular}{|c|c|c|c|}
\hline Cobertura morta & Diâmetro (cm) & $\begin{array}{c}\text { Massa fresca } \\
\left(\text { g planta }^{-1}\right)\end{array}$ & $\mathbf{N}\left(\mathbf{g ~ k g}^{-1}\right)$ \\
\hline & \multicolumn{3}{|c|}{$1^{\circ}$ Ciclo de cultivo } \\
\hline Cana-de-açúcar (S. officinarum) & $28,7 b^{1}$ & $246,3 \mathrm{~b}$ & $29,8 \mathrm{~b}$ \\
\hline Bambu (Bambuza sp.) & $28,0 \mathrm{~b}$ & $263,2 \mathrm{~b}$ & $32,4 \mathrm{~b}$ \\
\hline Capim Cameroon (P. purpureum) & $28,7 \mathrm{~b}$ & $279,4 \mathrm{~b}$ & $28,2 \mathrm{c}$ \\
\hline Crotalária (C. juncea) & 31,7 a & 366,0 a & 35,3 a \\
\hline Eritrina (E. poeppigiana) & 31,6 a & 340,6 a & 35,8 a \\
\hline Gliricídia (G. sepium) & 31,6 a & 347,9 a & 38,8 a \\
\hline Guandu (C. cajan) & $29,5 b$ & 328,3 a & $32,3 \mathrm{~b}$ \\
\hline Mucuna cinza (M. pruriens) & 30,8 a & 315,8 a & 36,5 a \\
\hline \multirow[t]{2}{*}{ Controle } & $26,3 \mathrm{~b}$ & $210,5 \mathrm{~b}$ & $27,8 \mathrm{c}$ \\
\hline & \multicolumn{3}{|c|}{$2^{\circ}$ Ciclo de cultivo } \\
\hline Cana-de-açúcar (S. officinarum) & $20,8 b$ & $100,8 \mathrm{c}$ & $26,0 \mathrm{~b}$ \\
\hline Bambu (Bambuza sp.) & 25,2 a & $152,3 \mathrm{~b}$ & $25,8 \mathrm{~b}$ \\
\hline Capim Cameroon (P. purpureum) & $21,4 \mathrm{~b}$ & $80,0 \mathrm{c}$ & $25,2 \mathrm{~b}$ \\
\hline Crotalária (C. juncea) & 26,8 a & 225,0 a & 30,3 a \\
\hline Eritrina (E. poeppigiana) & $28,1 \mathrm{a}$ & 214,5 a & $29,0 \mathrm{a}$ \\
\hline Gliricídia (G. sepium) & $28,5 \mathrm{a}$ & 205,7 a & 29,3 a \\
\hline Guandu (C. cajan) & $27,5 \mathrm{a}$ & 212,8 a & 29,2 a \\
\hline Mucuna cinza (M. pruriens) & $25,5 \mathrm{a}$ & 202,9 a & $28,0 \mathrm{a}$ \\
\hline Controle & $18,8 \mathrm{~b}$ & $103,6 \mathrm{c}$ & $23,7 \mathrm{~b}$ \\
\hline
\end{tabular}

${ }^{1}$ Para cada ciclo, médias seguidas da mesma letra, nas colunas, não diferem entre si pelo teste de Scott-Knott $(\mathrm{p}<0,05)$ (for each cultivation cycle, means followed by the same letter, in the column, do not differ from each other by Scott-Knott test, $\mathrm{p}<0.05$ ).

cola. Por outro lado, o fato do bambu apresentar relação $\mathrm{C} / \mathrm{N}$ intermediária entre as leguminosas e as demais gramíneas avaliadas pode amenizar problemas indicados por outros autores (Espindola et al., 2006) quanto à imobilização de nitrogênio em solos onde se adicionam resíduos de gramíneas.

O estudo evidenciou efeitos benéficos de coberturas do solo com resíduos de leguminosas e bambu, na produção orgânica de alface. Os benefícios trazidos pelas coberturas mortas de leguminosas resultaram, principalmente, da disponibilização de nitrogênio para a cultura, liberado através da acelerada decomposição dos resíduos. Independente da sua composição, todas as coberturas mortas foram eficientes no controle da vegetação espontânea.

\section{REFERÊNCIAS}

AITA C; GIACOMINI SJ. 2003. Decomposição e liberação de nitrogênio de resíduos culturais de plantas de cobertura de solo solteiras e consorciadas. Revista Brasileira de Ciência do Solo 27: 601-612.
AMBROSANO EJ; TRIVELIN PCO; CANTARELLA H; AMBROSANO GMB; MURAOKA T. 2003. Nitrogen mineralization in soils amended with sunnhemp, velvet bean and common bean residues. Scientia Agricola 60: 133-137.

ANDERSON JM; INGRAM JSI. 1989. Tropical soil biology and fertility: a handbook of methods. Wallingford: CAB International. $171 \mathrm{p}$.

BARRADAS CAA; FREIRE LR; ALMEIDA DL; DE-POLLI H. 2001. Comportamento de adubos verdes de inverno na região serrana fluminense. Pesquisa Agropecuária Brasileira 36: 1461-1468.

BREMNER JM; MULVANEY CS. 1982. Nitrogen total. In: PAGE AL. (ed). Methods of soil analysis. Part 2. 2.ed. Madison: SSSA. p.595-624.

CADAVID LF; EL-SHARKAWY MA; ACOSTA A; SÁNCHEZ T. 1998. Long-term effects of mulch, fertilization and tillage on cassava growth in sandy soils in Northern Colombia. Field Crops Research 57: 45-56.

CONSTANTIN J. 2001. Métodos de manejo. In: OLIVEIRA JUNIOR RS; CONSTANTIN J. (eds). Plantas daninhas e seu manejo. Guaíba: Agropecuária. p.103-121.

CONSTANTINIDES M; FOWNES JH. 1994. Nitrogen mineralization from leaves and litter of tropical plants: relationship to nitrogen, lignin and soluble polyphenol concentrations. Soil Biology and Biochemistry 26: 49-55. 
CORRÊA JC. 2002. Efeito de sistemas de cultivo na estabilidade de agregados de um Latossolo Vermelho-Amarelo em Querência, MT. Pesquisa Agropecuária Brasileira 37: $203-$ 209.

CORREIA MEF; ANDRADE AG. 1999. Formação de serapilheira. In: SANTOS GA; CAMARGO FAO. (eds). Fundamentos da matéria orgânica do solo: ecossistemas tropicais e subtropicais. Porto Alegre: Gênesis. p.97-225.

CORREIA NM; DURIGAN JC. 2004. Emergência de plantas daninhas em solo coberto com palha de cana-de-açúcar. Planta Daninha 22: 11-17.

EMBRAPA - Empresa Brasileira de Pesquisa Agropecuária. Centro Nacional de Pesquisa de Solos. 1997. Manual de métodos de análise de solo. Rio de Janeiro. 212p.

ERASMO EAL; AZEVEDO WR; SARMENTO RA; CUNHA AM; GARCIA SLR. 2004. Potencial de espécies utilizadas como adubo verde no manejo integrado de plantas daninhas. Planta Daninha 22: 337-342.
ESPINDOLA JAA; GUERRA JGM; ALMEIDA DL; TEIXEIRA MG; URQUIAGA S. 2006. Decomposição e liberação de nutrientes acumulados em leguminosas herbáceas perenes consorciadas com bananeira. Revista Brasileira de Ciência do Solo 30: 321-328.

GLIESSMAN SR. 2001. Agroecologia: processos ecológicos em agricultura sustentável. 2.ed. Porto Alegre: Editora da Universidade. 653p.

MacLEAN RH; LITSINGER JA; MOODY K; WATSON AK; LIBETARIO EM. 2003. Impact of Gliricidia sepium and Cassia spectabilis hedgerows on weeds and insect pests of upland rice. Agriculture, Ecosystems and Environment 94: 275-288.

MATTA-MACHADO RP; NEELY CL; CABRERA ML. 1994. Plant residue decomposition and nitrogen dynamics in an alley cropping and an annual legume-based cropping system. Communications in Soil Science and Plant Analysis 26: 1565-1570.

McDONAGH JF; TOOMSAN B; LIMPINUNTANA V; GILLER KE. 1995. Grain legumes and green manures as pre-rice crops in Northeast Thailand. II. Residue decomposition. Plant and Soil 177: 127-136.
PADOVAN MP; ALMEIDA DL; GUERRA JGM; RIBEIRO RLD; NDIAYE A. 2002. Avaliação de cultivares de soja, sob manejo orgânico, para fins de adubação verde e produção de grãos. Pesquisa Agropecuária Brasileira 37: 1705-1710.

PIRES NM; OLIVEIRA VR. 2001. Alelopatia. In: OLIVEIRA JUNIOR RS; CONSTANTIN J. (eds). Plantas daninhas e seu manejo. Guaíba: Agropecuária. p.145-185.

SMOLIKOWSKI B; PUIG H; ROOSE E. 2001. Influence of soil protection techniques on runoff, erosion and plant production on semiarid hillsides of Cabo Verde. Agriculture, Ecosystems and Environment 87: 67-80.

VAN SOEST PJ; WINE RH. 1968. Determination of lignin and cellulose in acid detergent fiber with permanganate. Journal of the Association of Official Analytical Chemists 51:780-785. 